\title{
SCALAR AND VECTOR VALUED PREMEASURES
}

\author{
M. K. NAYAK AND T. P. SRINIVASAN 1
}

ABSTRACT. A real valued (respectively Banach space valued) set-function on a lattice of sets extends to a $\sigma$-additive measure on a $\sigma$ field provided it is finitely additive tight, continuous at $\varnothing$ and has a bounded (respectively conditionally weakly compact) range.

In an earlier paper [2] with J. L. Kelley, we showed that a nonnegative valued function $\mu$ on a lattice $\mathcal{Q}$ of sets is a premeasure, meaning that it extends to a countably additive measure on a $\delta$ ring $\supset \mathbb{Q}$, provided $\mu$ is tight and continuous at $\varnothing$. The extension of the theorem to the case of real valued $\mu$ (not necessarily nonnegative valued) bugged us for sometime. The real valued case differs from the nonnegative case in that in the latter, for each monotonic increasing sequence $\left\{A_{n}\right\}$ of members of $\mathbb{Q}$, the sequence $\left\{\mu\left(A_{n}\right)\right\}_{n}$ is monotonic increasing and therefore has a limit, while for arbitrary real values, the existence of limits for such sequences is far from being clear. We show in this paper that the theorem indeed is true in the real valued case as well. An obvious additional hypothesis is necessary, namely that $\mu$ is locally bounded, i.e. $\forall A \in \mathbb{Q}$, $\operatorname{Sup}\{\mu(B) / B \in \mathbb{Q}, B \subseteq A\}<\infty$. (This is trivially true in case the values of $\mu$ are nonnegative.) Explicitly we have the following.

Theorem 1. A real valued $\mu$ on a lattice $\mathfrak{Q}$ of sets extends to a count ably additive measure on a $\delta$ ring (respectively $\sigma$-field) containing $\mathfrak{A}$ provided $\mu$ is

(i) locally bounded (respectively bounded),

(ii) finitely additive,

(iii) continuous at $\varnothing$,

(iv) tight.

In the above theorem we use a weaker definition for tightness than in

Presented to the Society, January 18, 1974; received by the editors October 8, 1973.

AMS (MOS) subject classifications (1970). Primary 28A45; Secondary 28A10.

Key words and phrases. Tight, modular, continuous at $\varnothing$. sas.

${ }^{1}$ This research was supported by grant $3440-5038$ from the University of Kan-

Copyright $\odot 1975$, American Mathematical Society 
[2], keeping in mind its adaptation to the vector valued case. When $\mu$ is nonnegative valued, conditions (i), (ii) and (iv) are all implied by the form of tightness assumed in [2].

The main point of the proof of Theorem 1 is that $\mu$ decomposes as usual as a difference, $\mu^{+}-\mu^{-}$, of nonnegative valued functions, where both $\mu^{+}$and $\mu^{-}$preserve all the properties of $\mu$ : finite additivity, continuity at $\varnothing$, and tightness. This is established in Steps $\mathrm{I}-\mathrm{V}$ below. Then the nonnegative valued case of the theorem proved in [2] applies to $\mu^{+}$and $\mu^{-}$and yields the theorem for $\mu$.

From the real valued case, a corresponding result follows for functions $\mu$ with values in a Banach space with or without the use of known theorems on extension [3]. The precise theorem in case of Banach space valued $\mu$ is as follows:

Theorem 2. A Banach space valued $\mu$ on a lattice $\mathbb{Q}$ of sets extends to a countably additive measure on a o-field containing $\mathbb{Q}$ with values in the Banach space provided $\mu$ is

(i) finitely additive,

(ii) continuous at $\varnothing$,

(iii) tight, and

(iv) $\mu[\mathfrak{Q}]$ is conditionally weakly compact.

We outline the proofs below. We need to spell out some definitions. Let $\mu$ be defined on a family $\mathscr{A}$ of sets with values in a Banach space $E$; we always assume that $\varnothing \in \mathbb{Q}$ and $\mu(\varnothing)=0$.

$\mu$ is said to be continuous at $\varnothing$ iff for each decreasing sequence $\left\{A_{n}\right\}_{n}$ of members of $\mathscr{Q}$ with $\bigcap_{n} A_{n}=\varnothing$, we have $\lim _{n} \mu\left(A_{n}\right)=0$.

$\mu$ is said to be tight iff for every pair of sets $A^{\prime}, A^{\prime \prime}$ in $\mathscr{G}$ such that $A^{\prime} \supseteq A^{\prime \prime}$, and for arbitrary $\epsilon>0$ there exists a set $A \in \mathbb{C}, A \subseteq A^{\prime}-A^{\prime \prime}$ such that $\left\|\mu\left(A^{\prime}\right)-\mu\left(A^{\prime \prime}\right)-\mu(A)\right\|<\epsilon$. (If the values of $\mu$ are real or complex scalars, the norm above is, of course, the absolute value.)

Proof of Theorem 1. Let $\mu^{+}, \mu^{-}$be defined on $\mathbb{A}$ by

$$
\mu^{+}\left(A^{\prime}\right)=\operatorname{Sup}\left\{\mu(A) / A \subseteq A^{\prime}, A \in \mathbb{P}\right\}
$$

and

$$
\mu^{-}\left(A^{\prime}\right)=-\inf \left\{\mu(A) / A \subseteq A^{\prime}, A \in \mathbb{Q}\right\} \text { for any } A^{\prime} \in \mathbb{Q} \text {. }
$$

Then $\mu^{+}$and $\mu^{-}$are finite and nonnegative because $\mu$ is assumed to be locally bounded. The proof is organized in the form of Steps I-V below. We note that $\mu^{-}=(-\mu)^{+}$. Consequently, the assertions on $\mu^{-}$in the following steps follow from the corresponding facts for $\mu^{+}$. 
Step I. $\mu=\mu^{+}-\mu^{-}$.

Proof. Let $\epsilon>0$ and let $A$ be any set in $\mathcal{Q}$. Then there exists $A_{1} \epsilon$ Q, $A_{1} \subseteq A$ such that

$$
\mu^{+}(A) \leq \mu\left(A_{1}\right)+\epsilon / 2 .
$$

Since $\mu$ is tight, there exists $A_{2} \in \mathbb{Q}, A_{2} \subseteq A-A_{1}$ such that

$$
\mu\left(A_{1}\right)+\mu\left(A_{2}\right) \leq \mu(A)+\epsilon / 2 .
$$

By $(\mathrm{I} .1)$ and (I.2)

$$
\begin{aligned}
\mu^{+}(A)-\mu^{-}(A) & \leq \mu\left(A_{1}\right)+\mu\left(A_{2}\right)+\epsilon / 2 \quad\left(\text { since }-\mu^{-}(A) \leq \mu\left(A_{2}\right)\right) \\
& \leq \mu(A)+\epsilon .
\end{aligned}
$$

Applying (I.3) to $-\mu$ instead of $\mu$, we get

$$
\mu^{+}(A)-\mu^{-}(A) \geq \mu(A)-\epsilon .
$$

Hence the result follows from (I.3) and (I.4).

Step II. $\mu^{+}, \mu^{-}$are finitely additive.

Proof. Let $\epsilon>0$ be chosen. Given any two disjoint sets $A_{1}, A_{2}$ in Q⿱ , by a suitable choice of $A_{11}: A_{21} \in \mathbb{Q}$ such that $A_{11} \subseteq A_{1}, A_{21} \subseteq A_{2}$, we have

$$
\begin{aligned}
\mu^{+}\left(A_{1}\right)+\mu^{+}\left(A_{2}\right) & \leq \mu\left(A_{11}\right)+\mu\left(A_{21}\right)+\epsilon \\
& =\mu\left(A_{11} \cup A_{21}\right)+\epsilon \leq \mu^{+}\left(A_{1} \cup A_{2}\right)+\epsilon .
\end{aligned}
$$

On the other hand there exists $\bar{A} \subseteq A_{1} \cup A_{2}$ such that

$$
\begin{aligned}
\mu^{+}\left(A_{1} \cup A_{2}\right) & \leq \mu(\bar{A})+\epsilon=\mu\left(\bar{A} \cap A_{1}\right)+\mu\left(\bar{A} \cap A_{2}\right)+\epsilon \\
& \leq \mu^{+}\left(A_{1}\right)+\mu^{+}\left(A_{2}\right)+\epsilon .
\end{aligned}
$$

It follows that $\mu^{+}\left(A_{1} \cup A_{2}\right)=\mu^{+}\left(A_{1}\right)+\mu^{+}\left(A_{2}\right)$.

Step III. $\mu^{+}, \mu^{-}$are tight.

Proof. Consider any pair of sets in $\mathbb{Q}$ such that one contains the other, say $A_{1}, A_{2} \in \mathbb{Q}, A_{1} \supseteq A_{2}$. Given $\epsilon>0$, there exists $A_{3} \in \mathbb{Q}$ such that $A_{3}$ $\subseteq A_{1}-A_{2}$ and

$$
\operatorname{Sup}\left\{\mu(A) / A \subseteq A_{1}-A_{2}\right\} \leq \mu\left(A_{3}\right)+\epsilon / 3 .
$$

This implies that any set $A$ disjoint with $A_{3}$ and lying in $A_{1}-A_{2}$ cannot have a measure larger than $\epsilon / 3$. Otherwise $A \cup A_{3}$ will have a measure larger than the supremum on the left-hand side of (1), which is impossible.

Let $A_{4} \subseteq A_{1}, A_{4} \in \mathbb{P}$, be so chosen that 
(III. 2)

$$
\mu^{+}\left(A_{1}\right) \leq \mu\left(A_{4}\right)+\epsilon / 3
$$

$$
=\mu\left(A_{4} \cap\left(A_{2} \cup A_{3}\right)\right)+\left(\mu\left(A_{4}\right)-\mu\left(A_{4} \cap\left(A_{2} \cup A_{3}\right)\right)\right)+\epsilon / 3 .
$$

By tightness we can choose $A \in \mathbb{Q}$ contained in $A_{4}-\left(A_{4} \cap\left(A_{2} \cup A_{3}\right)\right)$ so that $\mu(A)$ differs from $\mu\left(A_{4}\right)-\mu\left(A_{4} \cap\left(A_{2} \cup A_{3}\right)\right)$ by less than $\epsilon / 3$. By our remark above, $\mu(A) \leq \epsilon / 3$. It follows that the difference $\mu\left(A_{4}\right)-$ $\mu\left(A_{4} \cap\left(A_{2} \cup A_{3}\right)\right)$ is itself less than $2 \epsilon / 3$. Consequently from (III.2),

$$
\begin{gathered}
\mu^{+}\left(A_{1}\right)<\mu\left(A_{4} \cap\left(A_{2} \cup A_{3}\right)\right)+\epsilon \\
\leq \mu^{+}\left(A_{2} \cup A_{3}\right)+\epsilon=\mu^{+}\left(A_{2}\right)+\mu^{+}\left(A_{3}\right)+\epsilon ; \\
\mu^{+}\left(A_{1}\right)-\mu^{+}\left(A_{2}\right)<\mu^{+}\left(A_{3}\right)+\epsilon .
\end{gathered}
$$

The reverse inequality is obvious because $\mu^{+}$is monotone and finitely additive. Tightness of $\mu^{+}$(and therefore of $\mu^{-}$) follows.

Step IV. $\mu$ is modular; i.e. for every pair $A_{1}, A_{2} \in \mathbb{T}$

$$
\mu\left(A_{1} \cup A_{2}\right)+\mu\left(A_{1} \cap A_{2}\right)=\mu\left(A_{1}\right)+\mu\left(A_{2}\right) .
$$

Proof. Since $\mu=\mu^{+}-\mu^{-}$, it suffices to check that $\mu^{+}$(and therefore $\mu^{-}$) is modular. In turn (see [1]) it suffices to show that for $A_{1}, A_{2} \in \mathbb{Q}$, $A_{1} \supseteq A_{2}, \mu^{+}\left(A_{1}\right)-\mu^{+}\left(A_{2}\right)$ depends only on the difference $A_{1}-A_{2} \cdot$. This is immediate because, as a result of tightness and monotonicity of $\mu^{+}$,

$$
\mu^{+}\left(A_{1}\right)-\mu^{+}\left(A_{2}\right)=\operatorname{Sup}\left\{\mu^{+}(A) / A \subseteq A_{1}-A_{2}, A \in \mathbb{C}\right\} .
$$

Step V. $\mu^{+}, \mu^{-}$are continuous at $\varnothing$.

Proof. Let $\left\{A_{n}\right\}_{n}$ be a decreasing sequence in $\mathbb{Q}$ such that $\bigcap^{\infty} A_{n}=$ $\varnothing$. Let $\epsilon>0$ be chosen. A sequence $\left\{B_{n}\right\} \subset \mathbb{Q}, B_{n} \subseteq A_{n}, \forall n$ can be chosen such that

$$
\mu^{+}\left(A_{n}\right) \leq \mu\left(B_{n}\right)+\epsilon / 2^{n+1} .
$$

This means that any set contained in $A_{n}$ and disjoint with $B_{n}$ cannot have a measure larger than $\epsilon / 2^{n+1}$.

For any integer $n$ and any $k<n$, we can choose, by tightness, an $A \in \mathbb{Q}, A$ contained in

$$
\left(\left(B_{n} \cap\left(B_{1} \cap \cdots \cap B_{k-1}\right)\right) \cup B_{k}\right)-B_{k}
$$

so that $\mu(A)$ differs from

$$
\mu\left(\left(B_{n} \cap\left(B_{1} \cap B_{2} \cap \cdots \cap B_{k-1}\right)\right) \cup B_{k}\right)-\mu\left(B_{k}\right)
$$


by at most $\epsilon / 2^{k+1}$. By our remark above, $\mu(A) \leq \epsilon / 2^{k+1}$. It follows that for any $n$ and $k<n$

$$
\mu\left(\left(B_{n} \cap\left(B_{i} \cap \cdots \cap B_{k-1}\right)\right) \cup B_{k}\right)-\mu\left(B_{k}\right) \leq \epsilon / 2^{k} .
$$

Then we claim that for all $m$,

$$
\mu^{+}\left(A_{n}\right) \leq \mu\left(B_{1} \cap \cdots \cap B_{n}\right)+\epsilon / 2+\cdots+\epsilon / 2^{n} .
$$

For $n=1$, this immediately follows from (V.1). Next,

$$
\begin{aligned}
\mu^{+}\left(A_{2}\right) \leq \mu\left(B_{2} \cap B_{1}\right)+\mu\left(B_{2} \cup B_{1}\right)-\mu\left(B_{1}\right)+\epsilon / 2^{2} \\
\quad \text { (because of (V.1) and modularity of } \mu) \\
\leq \mu\left(B_{1} \cap B_{2}\right)+\epsilon / 2+\epsilon / 2^{2} \quad(\text { by (V.2)). }
\end{aligned}
$$

Similarly for $n=3$,

$$
\begin{aligned}
\mu^{+}\left(A_{3}\right) & \leq \mu\left(B_{3} \cap B_{1}\right)+\mu\left(B_{3} \cup B_{1}\right)-\mu\left(B_{1}\right)+\epsilon / 2^{3} \\
& \leq \mu\left(B_{3} \cap B_{1}\right)+\epsilon / 2+\epsilon / 2^{3} \quad(\text { by }(\mathrm{V} .2)) \\
& =\mu\left(B_{3} \cap B_{1} \cap B_{2}\right)+\mu\left(\left(B_{3} \cap B_{1}\right) \cup B_{2}\right)-\mu\left(B_{2}\right)+\epsilon / 2+\epsilon / 2^{3}
\end{aligned}
$$

(because $\mu$ is modular)

$$
\leq \mu\left(B_{1} \cap B_{2} \cap B_{3}\right)+\epsilon / 2+\epsilon / 2^{2}+\epsilon / 2^{3} \text { (by (V.2)). }
$$

Continuing the above process, we see that (V.3) holds good for all values of $n$. As $\bigcap_{n} B_{n}=\varnothing$ and $\mu$ is continuous at $\varnothing$, it follows from (V.3) that $\lim _{n} \mu^{+}\left(A_{n}\right)=0$.

We have shown that $\mu^{+}$and $\mu^{-}$satisfy the conditions for a premeasure set out in [2]. Further, they can be extended as measures on the $\sigma$-field generated by $Q$ in case $\mu$ is bounded instead of being locally bounded. The corresponding conclusion for $\mu$ follows.

We can derive Theorem 2 fromTheorem 1 using direct arguments. But we shall make use of a known extension theorem [3] and argue as follows. For each $x^{*}$ in $E^{*}$, the scalar function $x^{*} \mu$ is bounded, finitely additive, continuous at $\varnothing$, and tight, and, therefore, can be extended as a scalar measure $\widehat{x^{*} \mu}$ on the ring $R$ generated by $\mathcal{Q}$. Each member of $R$ is a finite disjoint union $\bigcup_{i=1}^{n}\left(A_{i}-B_{i}\right)$, where for each $i, A_{i}, B_{i} \in \mathbb{Q}$, and $A_{i} \supset B_{i}$. For each $x^{*}$ in $E^{*}$ we have

$$
x^{*}\left(\sum_{i}\left[\mu\left(A_{i}\right)-\mu\left(B_{i}\right)\right]\right)=\widehat{x^{*} \mu}\left(\bigcup_{i} A_{i} \backslash B_{i}\right) \text {, }
$$


which shows that if we let

$$
\bar{\mu}\left(\bigcup_{i=1}^{n}\left(A_{i}-B_{i}\right)\right)=\sum_{i=1}^{n}\left(\mu\left(A_{i}\right)-\mu\left(B_{i}\right)\right),
$$

then $\bar{\mu}$ is a well-defined weakly countably additive function on $R$ extending $\mu$. Using tightness we see that $\mu[\mathfrak{Q}]$ is dense in $\bar{\mu}[R]$ so that the latter is still weakly conditionally compact. Now the known theorem on extension applies to $\bar{\mu}$ (see $[3$, p. 178]) and completes the proof.

\section{REFERENCES}

1. J. L. Kelley and T. P. Srinivasan, Pre-measures on lattices of sets, Math. Ann. 190 (1970/71), 233-241. MR 43 \# 4990.

2. J. L. Kelley, M. K. Nayak and T. P. Srinivasan, Pre-measures on lattices of sets. II, Sympos. on Vector Measures, Salt Lake City, Utah, 1972.

3. I. Kluvanek, Vector and operator valued measures and applications, (Proc. Sympos., Snowbird Resort, Alta, Utah, 1972), Academic Press, New York, 1973, p. 178. 66044

DEPARTMENT OF MATHEMATICS, UNIVERSITY OF KANSAS, LAWRENCE, KANSAS 